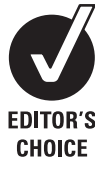

UMR745 INSERM, Université Paris Descartes, Faculté des Sciences Pharmaceutiques et Biologiques, Paris, France;

${ }^{2}$ Service de Biochimie et de Génétique Moléculaire, Hôpital Beaujon, AP-HP, Clichy, France: ${ }^{3}$ Service d'Hématologie Biologique, Hôpital Armand Trousseau, AP-HP, Paris, France; ${ }^{4}$ Service de Génétique, Centre hospitalier Félix Guyon, Bellepierre, Saint-Denis, France; ${ }^{5}$ Service de Dermatologie, Hôpital Hôtel Dieu, Nantes, France; ${ }^{6}$ Service d'Oncologie Pédiatrique, Hôpital Armand Trousseau, AP-HP, Paris, France; ${ }^{7}$ Service de Biochimie B Hormonale, Métabolique et Génétique, Hôpital Bichat Claude-Bernard, AP-HP, Paris, France; ${ }^{8}$ Service de Dermatologie, Hôpital Henri Mondor, AP-HP, Créteil, France

Correspondence to: Dr E Pasmant, UMR745 INSERM, Université Paris

Descartes, Faculté des Sciences Pharmaceutiques et Biologiques, 4 avenue de l'Observatoire, 75006, Paris, France; eric.pasmant@etu.univ-paris5.fr

Received 11 December 2008 Revised 6 February 2009 Accepted 11 February 2009 Published Online First 14 April 2009

\title{
SPRED1 germline mutations caused a neurofibromatosis type 1 overlapping phenotype
}

\author{
E Pasmant, ${ }^{1,2}$ A Sabbagh, ${ }^{1,2} \mathrm{~N}$ Hanna, ${ }^{1,2} \mathrm{~J}$ Masliah-Planchon, ${ }^{2}$ E Jolly, ${ }^{2}$ P Goussard, ${ }^{2}$ \\ P Ballerini, ${ }^{3}$ F Cartault, ${ }^{4}$ S Barbarot, ${ }^{5}$ J Landman-Parker, ${ }^{6}$ N Soufir, ${ }^{7}$ B Parfait, ${ }^{1,2}$ \\ $\mathrm{M}$ Vidaud, ${ }^{1,2} \mathrm{P}$ Wolkenstein, ${ }^{8} \mathrm{D}$ Vidaud, ${ }^{1,2} \mathrm{R}$ N F France
}

\begin{abstract}
Objective: Germline loss-of-function mutations in the SPRED1 gene have recently been identified in patients fulfilling the National Institutes of Health (NIH) diagnostic criteria for neurofibromatosis type 1 (NF1) but with no NF1 (neurofibromin 1) mutation found, suggesting a neurofibromatosis type 1-like syndrome.
\end{abstract}

Methods: 61 index cases with NF1 clinical diagnosis but no identifiable NF1 mutation were screened for SPRED1 mutation.

Results: We describe one known SPRED1 mutation (c.190C $>$ T leading to p.Arg64Stop) and four novel mutations (c.637C $>$ T leading to p.Gln213Stop, c.2T $>C$ leading to p.Met1Thr, c.46C $>$ T leading to p.Arg16Stop, and c.1048 1060del leading to p.Gly350fs) in five French families. Their NF1-like phenotype was characterised by a high prevalence of café-au-lait spots, freckling, learning disability, and an absence of neurofibromas and Lisch nodules in agreement with the original description. However, we did not observe Noonan-like dysmorphy. It is noteworthy that one patient with the p.Arg16Stop mutation developed a monoblastic acute leukaemia.

Conclusions: In our series, SPRED1 mutations occurred with a prevalence of $0.5 \%$ in NF1 patients and in $5 \%$ of NF1 patients displaying an NF1-like phenotype. SPRED1 mutated patients did not display any specific dermatologic features that were not present in NF1 patients, except for the absence of neurofibromas that seem to be a specific clinical feature of NF1. The exact phenotypic spectrum and the putative complications of this NF1 overlapping syndrome, in particular haematological malignancies, remain to be further characterised. NIH diagnostic criteria for NF1 must be revised in view of this newly characterised Legius syndrome in order to establish a specific genetic counselling.

Germline mutations in genes involved in the Rasmitogen activated protein kinase (MAPK) signalling pathway have been reported in several phenotypically overlapping syndromes with autosomal dominant transmission. ${ }^{1}$ These observations provided a unifying mechanism-Ras MAPK pathway constitutive activation-leading to neurocardio-facial-cutaneous (NCFC) syndromes (fig 1). These rare diseases include neurofibromatosis 1 (NF1, OMIM 162200), Noonan syndrome (NS, OMIM 163950), LEOPARD syndrome (LS, OMIM 151100), cardio-facio-cutaneous syndrome (CFC, OMIM 115150), and Costello syndrome (CS, OMIM 218040). Predisposition to malignancies is a known feature of NCFC syndromes with the exception of CFC. ${ }^{2}$
NF1 has a birth incidence of $\sim 1 / 3000$ and has been showed to be caused by autosomal dominant loss of function mutations of the NF1 (neurofibromin 1; NM 000267) gene in approximately $95 \%$ of patients fulfilling the National Institutes of Health (NIH) clinical diagnostic criteria. ${ }^{3-5}$ NF1 is located in 17q11.2 and contains 60 translated exons distributed over $\sim 350 \mathrm{~kb}$. Most of the NF1 mutations (90-95\%) are intragenic mutations (point mutation, splice mutation, small deletion, insertion, or duplication). The remaining NF1 mutations (5-10\%) are microdeletions encompassing the entire NF1 gene and its neighbouring genes. ${ }^{6}$ The protein encoded by NF1 (neurofibromin) is a Ras-GTPase activating protein (Ras-GAP) that acts as a negative regulator of the Ras-MAPK cascade.?

Germline dominant loss-of-function mutations in the SPRED1 (sprouty-related, EVH1 domain containing 1; NM_152594) gene have recently been identified in five unrelated families and seven additional patients fulfilling the NIH diagnostic criteria for NF1, although no NF1 mutation was found. ${ }^{8}$ Remarkably, their phenotype referred as neurofibromatosis type 1-like syndrome (NFLS, OMIM 611431) consisted of multiple café-au-lait spots, axillary freckling, and macrocephaly but without neurofibromas and Lisch nodules. The SPRED1 gene, located in 15q13.2, contains seven coding exons, and encodes the SPRED1 protein. Sprouty (SPRY1, $-2,-3$, and -4) and SPRED (SPRED1, -2 , and -3 ) proteins are an evolutionarily conserved family of membrane associated negative regulators of growth factor induced extracellular signal regulated kinases (ERK) activation. ${ }^{9}{ }^{10}$ SPRED1 specifically inhibits mitogen activated protein kinases (MAPK) signalling by suppressing Raf phosphorylation and activation. ${ }^{11}$

In the present study, we confirmed the existence of loss-of-function mutations of SPRED1 and the characterisation of a NCFC syndrome with an NF1 overlapping phenotype. We identified three nonsense mutations, one missense mutation, and one frameshift deletion of SPRED1 among 61 families fulfilling $\mathrm{NIH}$ diagnostic criteria for NF1 but with no identifiable NF1 mutation. We then developed a molecular diagnostic algorithm for individuals displaying a NF1like phenotype tailored to routine clinical practice.

\section{PATIENTS, MATERIALS, AND METHODS}

\section{Patients}

A database devoted to NF1 has been established in France thanks to a grant from the French Clinical Research Program (coordinator: Professor P Wolkenstein, Henri Mondor Hospital, Créteil, 
Figure 1 A schematic of Ras signalling. Germline mutations in key components of the Ras-mitogen activated protein kinase (MAPK) signalling pathway cause neurocardio-facial-cutaneous (NCFC) syndromes. NF1, neurofibromatosis 1 .

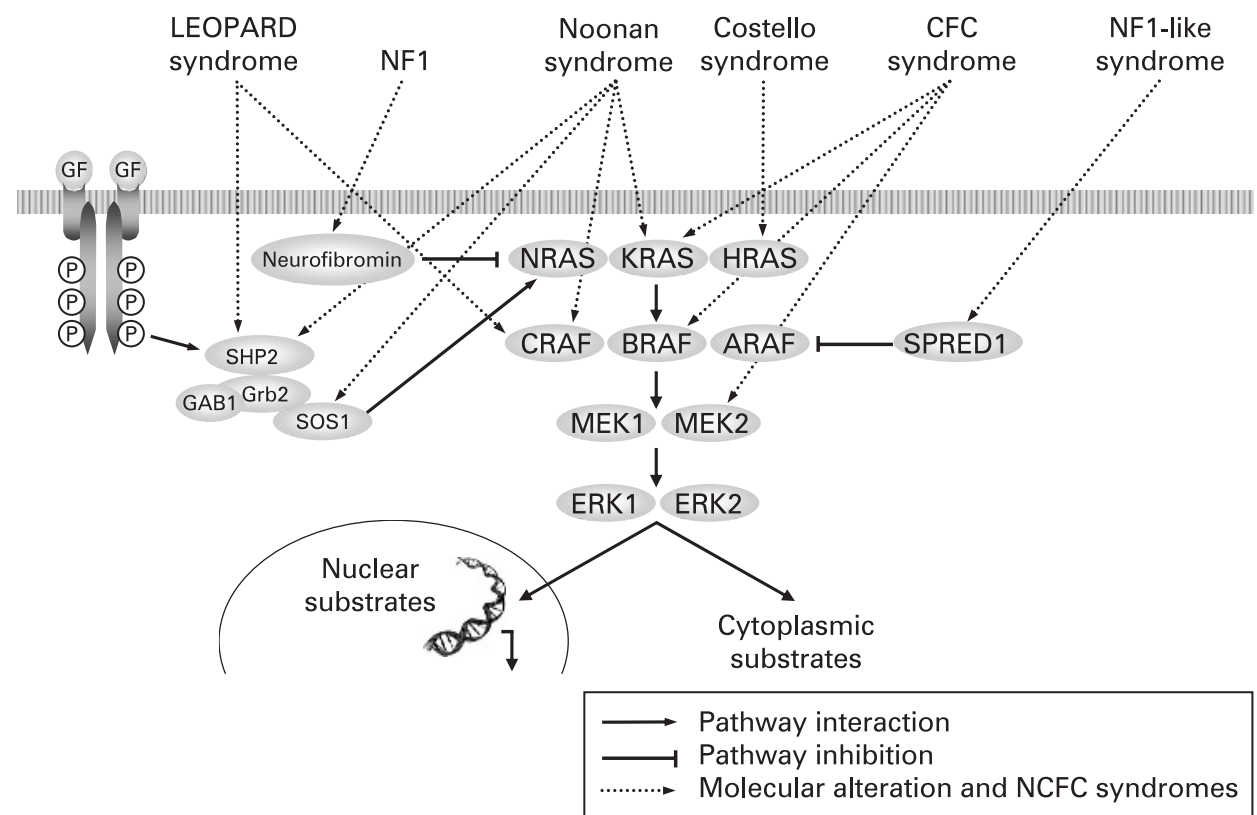

France). The collection consists of 561 families, including 1697 individuals among which 1083 were NF1 patients fulfilling the $\mathrm{NIH}$ diagnostic criteria. The phenotype of each patient was described in a standardised way and was coupled with a comprehensive analysis of the NF1 mutational spectrum including intragenic microsatellites polymorphisms study, NF1 sequencing at both RNA and DNA levels, and real-time polymerase chain reaction (PCR) based gene dosage (data not shown). Among the 561 NF1 index cases, an NF1 mutation was successfully identified in 512 individuals. The 49 NF1 index cases lacking NF1 mutations were selected as a first panel from this NF-France database to be screened for SPRED1 mutation.

A second panel of 12 NF1 clinically diagnosed index cases with no NF1 mutation was phenotypically selected from routine genetic counselling: their phenotypes lay within the NF1 spectrum but were distinctive, with a high prevalence of café-au-lait spots, axillary, and groin freckling, but absence of neurofibromas and Lisch nodules.

All blood samples drawn for nucleic acid (DNAs and RNAs) extraction were collected after informed consent.

\section{DNA and RNA extraction}

High molecular weight DNA was prepared by standard proteinase $\mathrm{K}$ digestion followed by phenol-chloroform extraction from whole blood leucocytes. Total RNA was extracted from whole blood leucocytes by using the acid-phenol guanidium method. The quality of the nucleic acids was determined by electrophoresis through agarose gels and staining with ethidium bromide (DNA and RNA), and the 18S and $28 \mathrm{~S}$ RNA bands were visualised under ultraviolet light (RNA).

The somatic leukaemia DNA was extracted from bone marrow of patient II:1 in family 4 (showing $85 \%$ of blastic cells) upon diagnosis of acute leukaemia.

\section{SPRED1 mutation screening}

We performed SPRED1 mutation screening at both RNA and genomic DNA levels. Mutational screening of SPRED1 was carried out on RNA through the following procedure. After reverse transcription with Superscript II RNase H-Reverse
Transcriptase (Invitrogen, Cergy-Pontoise, France), two overlapping PCR fragments were generated by Taqman PCR Core Reagent Kit (Applied Biosystems, Courtaboeuf, France). Mutation screening was performed with the ABI BigDye terminator sequencing kit (Applied Biosystems) on an ABI Prism 3130 automatic DNA sequencer (Applied Biosystems). Sequences were aligned with Seqscape analysis software (Applied Biosystems). The primer oligonucleotide sequences and PCR conditions are available on demand.

Genomic DNA was amplified with primers specific for SPRED1 coding exons and their IVS boundaries. The primer oligonucleotide sequences and PCR conditions are available upon request. PCR was performed with the Taqman PCR Core Reagent Kit (Applied Biosystems). Mutation screening was performed using bidirectional DNA sequencing of purified PCR products.

\section{SPRED1 real-time PCR based gene dosage}

In this method, quantitative values are obtained from the threshold cycle number at which the increase in the signal associated with exponential growth of PCR products begins to be detected by Applied Biosystems analysis software. The precise amount of genomic DNA added to each reaction mix (based on optical density) and its quality (that is, lack of extensive degradation) are both difficult to assess. We therefore also quantified the $A L B$ gene (encoding albumin and mapping to chromosome region 4q11-q13) as an endogenous DNA control, and each sample was normalised on the basis of its $A L B$ content. The relative SPRED1 copy number was also normalised to a calibrator, or 1X sample, consisting of genomic DNA from a normal subject. Final results, expressed as $\mathrm{N}$-fold differences in the target gene copy number relative to the $A L B$ gene and the calibrator, and termed "Ntarget", were determined as follows: Ntarget $=2^{(\Delta \text { Ctsample }-\Delta \text { Ctcalibrator })}$, where $\Delta \mathrm{Ct}$ values of the sample and calibrator are determined by subtracting the average $\mathrm{Ct}$ value of the target gene from the average Ct value of the $A L B$ gene. Given the target gene marker, samples with Ntarget values of 0.5 and 1.0 were considered deleted and normal, respectively. Primers for $A L B$, and SPRED1 gene dosage were chosen with the assistance of the computer program Oligo 4.0 (National Biosciences). SPRED1 exons 3, 4, and 8 were 
Figure 2 Pedigrees of families 1, 2, 3, 4, and 5. Squares and circles indicate males and females, respectively. Open symbols indicate unaffected individuals, filled symbols indicate affected individuals, arrows indicate propositus, and symbols with a slash indicate deceased family members.
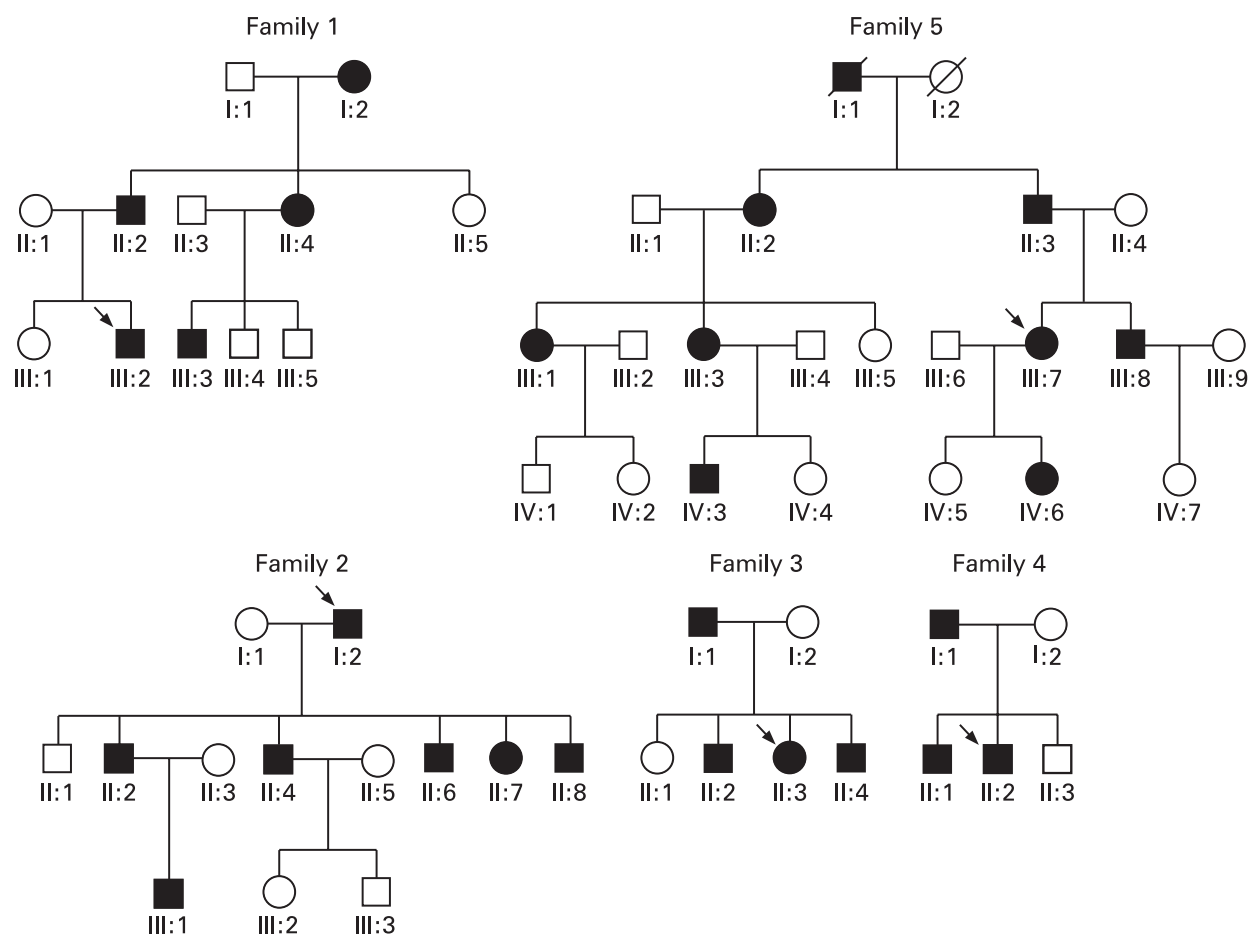

quantified to copy number. The primer oligonucleotide sequences and PCR conditions are available upon request. All PCR reactions were performed with an ABI Prism 7900 Sequence Detection System (Applied Biosystems) and the SYBR Green PCR Core Reagents kit (Applied Biosystems). Experiments were performed in triplicate for each data point.

\section{RESULTS}

\section{Germline DNAs molecular analysis}

Forty-nine index cases with no identifiable NF1 mutation from the French NF1 database were screened for mutation in the SPRED1 gene. Two SPRED1 heterozygous nonsense mutations were identified at both DNA and RNA levels: one known mutation (family 1: c.190C >T leading to p.Arg64Stop) and one novel mutation (family 2: c.637C > T leading to p.Gln213Stop) (fig 2).

SPRED1 mutational analysis of an additional set of 12 index cases selected from routine genetic counselling on the basis of their NF1-like phenotype (presence of café-au-lait spots and/or freckling and absence of neurofibroma and Lisch nodule), identified three novel heterozygous mutations: one missense mutation (family 3: c.2T $>$ C leading to p.Met1Thr), one nonsense mutation (family 4: c.46C $>\mathrm{T}$ leading to p.Arg16Stop), and one frame-shift deletion (family 5: c.1048_1060del leading to p.Gly350fs) (fig 2).

In families 1, 2, and 3, the SPRED1 mutations were present in all affected individuals and were absent in three tested unaffected relatives (family 1: individual II:1, family 2: I:1, and family 3: I:1) (table 1). In families 4 and 5 the propositus showed SPRED1 mutations.

The clinical features of SPRED1 tested individuals of families $1,2,3,4$, and 5 are displayed in table 1 .

\section{Molecular analysis of the somatic leukaemic cells of patient II:2 (family 4)}

In order to test the tumour suppressor gene hypothesis, SPRED1 molecular analysis was performed on the leukaemic cells DNA of patient II:2 of family 4. No somatic additional SPRED1 alteration (including point mutation and loss of heterozygosity) was observed.

\section{DISCUSSION}

The purpose of this study was to confirm the recently identified loss-of-function mutations of SPRED1 in a new NF1 overlapping syndrome $e^{8}$ (named NFLS) and to specify its phenotype and incidence. We confirmed one known mutation (p.Arg64Stop) described in the original paper of Brems et a ${ }^{8}$ and reported four novel mutations (p.Gln213Stop, p.Met1Thr, p.Arg16Stop, and p.Gly350fs) in five French families fulfilling NIH NF1 diagnostic criteria. Similar to the mutation p.Gln215Stop previously reported by Brems et $a l^{8}$ the nonsense mutation p.Gln213Stop found in family 2 also affected exon 7 of SPRED1. The missense mutation c.2T $>\mathrm{C}$ (leading to p.Met1Thr) identified at both DNA and RNA levels (ruling out a putative RNA decay) at the initiation codon in exon 2 abolished initiation of translation at the normal start site. Although the first new potential AUG codon downstream of the mutation at the position corresponding to methionine 21 could conceivably be used as an in-frame initiation codon, the loss of 20 amino acids from the Ena/vasodilator stimulated phosphoprotein (VASP) homology (EVH-1) N-terminal functional domain of SPRED1 would probably result in a nonfunctional protein. It is notable that most of the reported mutations of SPRED1 affect the EVH-1 domain. ${ }^{8}$ Nonetheless, the precise consequences of the p.Met1Thr mutation at the protein level remain to be determined by experimental means. The frame shift deletion p.Gly350fs affected the C-terminus SPROUTY-like (SPR) domain of SPRED1, like p.Ser383fs previously reported. ${ }^{8}$ These five mutations may result in a loss of function of SPRED1, known to inhibit the Ras-MAPK cascade, consistent with the concept that NCFC syndromes are caused by aberrant activation of this pathway.

The mechanism of inheritance in the two large families 1 and 2 (with five and seven affected and mutated individuals, respectively) was compatible with an autosomal dominant 


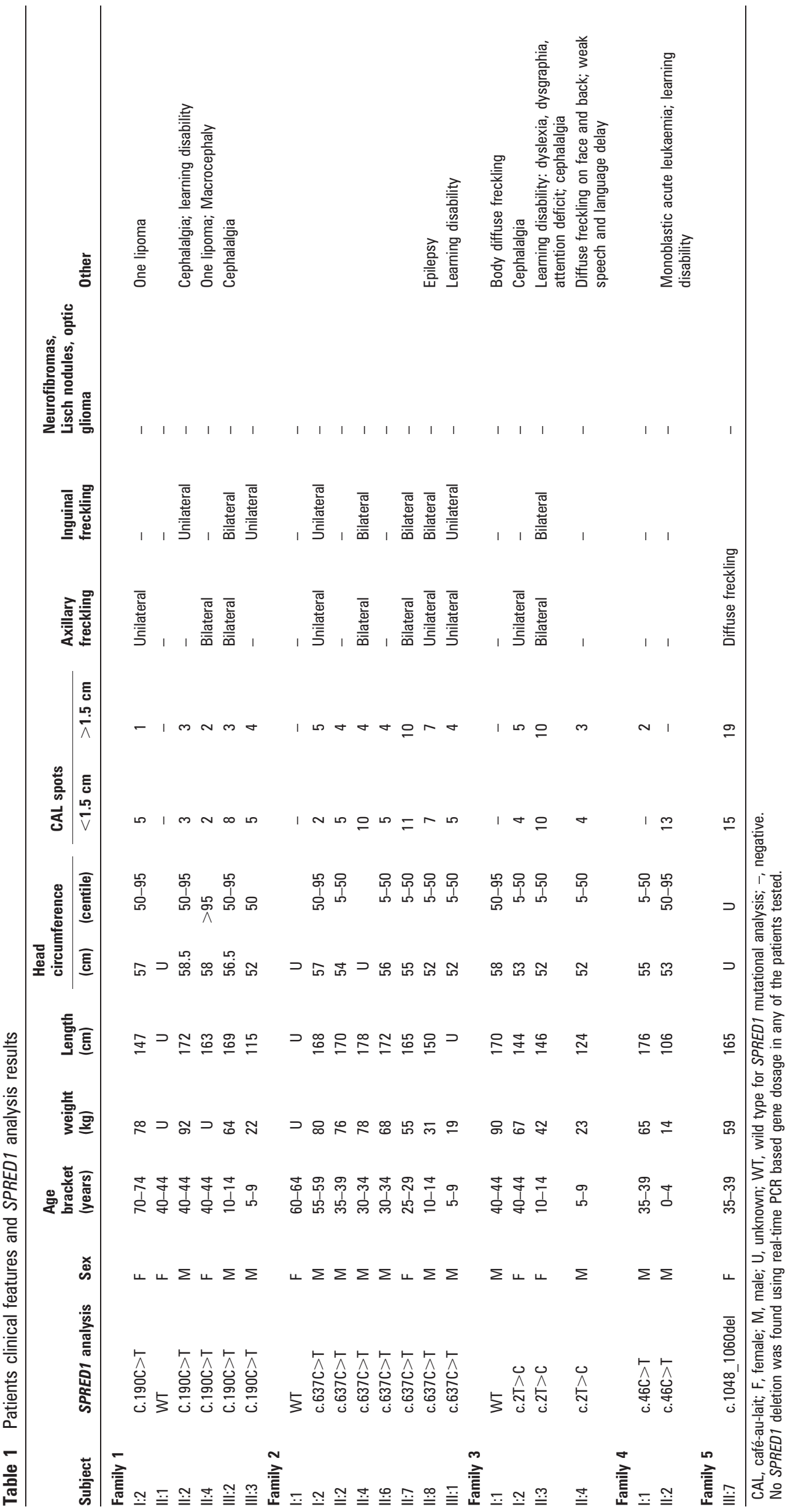


pattern with complete penetrance. The clinical features of patients with SPRED1 defects in our study (table 1) were quite similar to the ones described by Brems et al. ${ }^{8}$ The phenotype lay within the NF1 spectrum but was distinctive, with a high prevalence of café-au-lait spots and axillary and groin freckling and an absence of neurofibromas and Lisch nodules. Remarkably, some previously additional reported features were also found in the reported patients: learning disability was observed in four of the five reported families (families 1, 2, 3, and 4) and lipomas in one family (family 2). However, macrocephaly was found in only one individual and Noonanlike dysmorphy was not observed in any of the five families (table 1), contrary to the original description of Brems et al. ${ }^{8}$ Based on these observations, the designation "neurofibromatosis 1-like phenotype" does not seem appropriate because of the absence of neurofibroma. We therefore propose to rename the condition caused by heterozygous SPRED1 mutations as "Legius syndrome". The café-au-lait spots and freckling found in SPRED1 mutated patients in our study were similar to those found in NF1 patients with NF1 mutations. Therefore, NF1 and SPRED1 mutated patients could not be distinguished on the dermatologic features (aspect and number) of their café-au-lait spots and freckling. Moreover, learning disability seems to be a quite constant feature of this new syndrome. It is noteworthy that the behavioural deficits of Spred1-/ - and Spred1+/ - mice mimic the neurocognitive impairments in $N f 1+/-$ mice with a remarkably similar synaptic phenotype. ${ }^{12}$

Among the 561 NF1 index cases of the first panel (fulfilling the NIH diagnostic criteria for NF1), 91\% (512/561) showed an NF1 mutation, $0.5 \%(2 / 561)$ showed a SPRED1 mutation, and $8.5 \%(47 / 561)$ did not show mutation in neither SPRED1 nor NF1. These data confirmed that SPRED1 mutations are rare events that are likely to be underestimated because of their mild associated phenotype that may cause fewer medical consultations compared to NF1 cases with neurofibromas. The NF1-like phenotype (here defined as café-au-lait spots and/or freckling with no neurofibroma and no Lisch nodule) had a poor prevalence among all NF1 cases: among the 561 NF1 index cases, $8 \%(43 / 561)$ displayed an NF1-like phenotype, among which $79 \%$ (34/43) showed an NF1 mutation and 5\% (2/43) showed a SPRED1 mutation. Among the 49/561 (9\%) NF1 index cases with no identifiable NF1 mutation, 9/49 (18\%) index cases showed an NF1 like phenotype: six were isolated NF1 cases (only one NF1 individual recruited per family) and three were families with at least two recruited NF1 individuals. Among these three families, only two showed at least one postpubertal patient (neurofibromas usually develop in the late teens or early 20s). Interestingly, these two families were exactly those showing a SPRED1 mutation (family 1 and family 2). In light of these new observations, we adapted our molecular diagnostic strategy to routine clinical practice: post-pubertal patients with a family history of café-au-lait spots and/or freckling with neither neurofibromas nor Lisch nodule are now screened for mutation in SPRED1 before performing NF1 screening. Families 3,4 , and 5 satisfied these conditions.

It is noteworthy that patient II: 2 in family 4 developed a monoblastic acute leukaemia, suggesting a possible implication of SPRED1 germline mutation. SPRED1 is highly expressed in haematopoietic cells and negatively regulates haematopoiesis by suppressing stem cell factor (SCF) and interleukin 3 (IL3) induced ERK activation. ${ }^{13}$ Although rare, inherited predispositions to myeloid leukaemia have uncovered a critical role of hyperactive Ras signalling in normal myeloid growth and leukaemogenesis. ${ }^{14-16}$ Moreover, individuals with NS,
LEOPARD syndrome, and NF1 have a higher risk of haematological malignancies, including acute myeloid leukaemia (AML) and the rare disorder juvenile myelomonocytic leukaemia (JMML). ${ }^{217}$ With regards to these observations, SPRED1 seemed to be a good candidate gene for leukaemia predisposition. However, molecular analysis of the tumour DNA did not show additional somatic SPRED1 alteration (including point mutation and loss of heterozygosity), in contradiction to the Knudson two-hits model for tumour suppressor genes. Considering our observation and the data implicating hyperactive Ras in the pathogenesis of JMML, as well as the role of SPRED1 in modulating signals from haematopoietic growth factor receptors to Ras, it would be of interest to screen SPRED1 for mutation in the $\sim 20 \%$ of JMML cases showing no mutations in either KRAS, NRAS, PTPN11, or NF1 $1^{18}$ so as to confirm its putative implication in leukaemogenesis.

In conclusion, SPRED1 mutations were present in $0.5 \%$ of all NF1 cases and in 5\% of NF1 patients displaying an NF1like phenotype. SPRED1 mutated patients did not display specific dermatologic features not present in NF1 patients, except for the absence of neurofibromas that seem to be a specific clinical feature of NF1. NIH diagnostic criteria for NF1 must now take into account these new data to actualise guidelines and to establish a specific genetic counselling. Further studies will be necessary to characterise the frequency of SPRED1 mutations, the exact phenotypic spectrum (including learning disability), and the putative complications (in particular haematological malignancies) of this Legius syndrome caused by a new mechanism through which RasMAPK signalling is deregulated. These findings also emphasise the need to test families with NF1-like phenotype with no NF1 and SPRED1 mutations for additional genes involved in the Ras-MAPK pathway.

Acknowledgements: We thank the patients and their parents for participation.

Funding: This work was supported in part by grants by Association Neurofibromatoses et Recklinghausen, Ligue Française Contre les Neurofibromatoses the Clinical Research programme (PHRC 2002), INSERM (NF1GeneModif project) and Ministère de l'Enseignement Supérieur et de la Recherche.

Competing interests: None.

\section{REFERENCES}

1. Bentires-Alj M, Kontaridis MI, Neel BG. Stops along the RAS pathway in human genetic disease. Nat Med 2006;12:283-5.

2. Schubbert S, Shannon K, Bollag G. Hyperactive Ras in developmental disorders and cancer. Nat Rev Cancer 2007;7:295-308.

3. Ferner RE, Huson SM, Thomas N, Moss C, Willshaw H, Evans DG, Upadhyaya M, Towers R, Gleeson M, Steiger C, Kirby A. Guidelines for the diagnosis and management of individuals with neurofibromatosis 1. J Med Genet 2007:44:81-8.

4. National Institutes of Health. National Institutes of Health Consensus Development Conference Statement. Neurofibromatosis. Arch Neurol Chicago 1988;45:575-8.

5. Messiaen LM, Callens T, Mortier G, Beysen D, Vandenbroucke I, Van Roy N, Speleman F, Paepe AD. Exhaustive mutation analysis of the NF1 gene allows identification of $95 \%$ of mutations and reveals a high frequency of unusual splicing defects. Hum Mutat 2000;15:541-55.

6. Kluwe L, Siebert R, Gesk S, Friedrich RE, Tinschert S, Kehrer-Sawatzki H, Mautner VF. Screening 500 unselected neurofibromatosis 1 patients for deletions of the NF1 gene. Hum Mutat 2004;23:111-6.

7. Xu GF, O'Connell P, Viskochil D, Cawthon R, Robertson M, Culver M, Dunn D, Stevens J, Gesteland R, White R, Weiss R. The neurofibromatosis type 1 gene encodes a protein related to GAP. Cell 1990;62:599-608.

8. Brems H, Chmara M, Sahbatou M, Denayer E, Taniguchi K, Kato R, Somers R, Messiaen L, De Schepper S, Fryns JP, Cools J, Marynen P, Thomas G, Yoshimura A, Legius E. Germline loss-of-function mutations in SPRED1 cause a neurofibromatosis 1like phenotype. Nat Genet 2007;39:1120-6.

9. Bundschu K, Walter U, Schuh K. Getting a first clue about SPRED functions. Bioessays 2007;29:897-907.

10. Kim HJ, Bar-Sagi D. Modulation of signalling by Sprouty: a developing story. Nat Rev Mol Cell Biol 2004;5:441-50. 
11. Wakioka T, Sasaki A, Kato R, Shouda T, Matsumoto A, Miyoshi K, Tsuneoka M, Komiya S, Baron R, Yoshimura A. Spred is a Sprouty-related suppressor of Ras signalling. Nature 2001;412:647-51.

12. Denayer E, Ahmed T, Brems H, Van Woerden G, Borgesius NZ, Callaerts-Vegh Z, Yoshimura A, Hartmann D, Elgersma Y, D'Hooge R, Legius E, Balschun D. Spred1 is required for synaptic plasticity and hippocampus-dependent learning. J Neurosci 2008;28:14443-9.

13. Nonami A, Kato R, Taniguchi K, Yoshiga D, Taketomi T, Fukuyama S, Harada M, Sasaki A, Yoshimura A. Spred-1 negatively regulates interleukin-3-mediated ERK/mitogen-activated protein (MAP) kinase activation in hematopoietic cells. J Biol Chem 2004;279:52543-51.

14. Steelman LS, Abrams SL, Whelan J, Bertrand FE, Ludwig DE, Bäsecke J, Libra M, Stivala F, Milella M, Tafuri A, Lunghi P, Bonati A, Martelli AM, McCubrey JA. Contributions of the Raf/MEK/ERK, PI3K/PTEN/Akt/mTOR and Jak/STAT pathways to leukemia. Leukemia 2008;22:686-707.
15. Renneville A, Roumier C, Biggio V, Nibourel O, Boissel N, Fenaux P, Preudhomme C Cooperating gene mutations in acute myeloid leukemia: a review of the literature. Leukemia 2008;22:915-31.

16. Bowen DT, Frew ME, Hills R, Gale RE, Wheatley K, Groves MJ, Langabeer SE, Kottaridis PD, Moorman AV, Burnett AK, Linch DC. RAS mutation in acute myeloid leukemia is associated with distinct cytogenetic subgroups but does not influence outcome in patients younger than 60 years. Blood 2005;106:2113-9.

17. Tartaglia M, Gelb BD. Noonan syndrome and related disorders: genetics and pathogenesis. Annu Rev Genomics Hum Genet 2005;6:45-68.

18. Loh ML, Vattikuti S, Schubbert S, Reynolds MG, Carlson E, Lieuw KH, Cheng JW Lee CM, Stokoe D, Bonifas JM, Curtiss NP, Gotlib J, Meshinchi S, Le Beau MM, Emanuel PD, Shannon KM. Mutations in PTPN11 implicate the SHP-2 phosphatase in leukemogenesis. Blood 2004;103:2325-31. 